\title{
The Application Comparison of bottom anchored cable-stayed cantilever scaffold in building assembly
}

\author{
LanYun Chen ${ }^{1, a}$, QuanWei Zhao ${ }^{2, b}$ \\ ${ }^{1}$ Jinhua Polytechnic, Jinhua, China \\ 2Jinhua Polytechnic,Jinhua,China

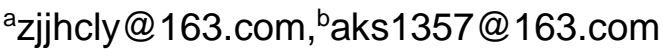

\begin{abstract}
Key word: Cantilever scaffolding; I-steel; bottom anchor; safety
Abstract.Scaffolding erection is an indispensable process in high-rise building construction, which directly affects the safety of building construction. The traditional cable-stayed cantilevered scaffold erected with cantilevered beam faces the problem that the prefabricated part of the building can not be anchored.It is urgent to find a new type of cable-stayed cantilever scaffold to solve the cantilevered problem. Through the application and comparison analysis, the bottom anchor cablestayed cantilever scaffolding can not only solve the problem of the traditional anchorage of the traditional cable-stayed cantilever beam, but also basically solve the hidden dangers of the external wall seepage, the safety performance of the outer frame and the structure safety of the traditional cable-stayed cantilever scaffold, at the same time save the amount of steel material and reduce the time of the demolition and repair. The economic benefits have been improved.
\end{abstract}

\section{Introduction}

At present, the cantilever scaffolding used in construction can be divided into cantilever type, oblique bracing type, bracing type and cable-stayed form according to the different force mode, while the most used in construction site is the cable-stayed cantilever scaffolding.Cantilever beam members at the bottom of traditional cable-stayed cantilever scaffolds (Figure 1,2),generally, it is I-steel, its inner end is fixed on the floor and the I-steel is anchored on the floor ${ }^{[1]}$. According to the length of the cantilever, there are two or three points, and the anchorage is made of 20 steel, which is made of $U$ type. It is pre buried in the concrete of the cast-in-place slab. After the installation of I-steel, the upper entrance of the steel bar is closed and welded.In recent years, the country has vigorously promoted the construction industrialization and assembly, and the construction industrialization and assembly are the important means to promote the energy saving and emission reduction, improve the quality of construction, and promote the transformation and upgrading of the construction industry.By building industrialization and closely relying on scientific and technological progress and technological innovation, we must say goodbye to the traditional mode of building production with high energy consumption, high pollution, low efficiency and low efficiency. The main assembly structure of building industrialization is PC laminated board and prefabricated staircase assembly.The implementation of building assembly is generally standard floor with prefabricated components, mainly PC laminated panels and fabricated staircases.In this way, the traditional cable-stayed cantilever scaffold can not be buried on the PC laminated plate by the method of holding the hoop in the beam and the cast-in-place slab, and even embedded in the PC laminated plate, it will cause a great damage to the PC component.Therefore, the traditional cantilever scaffolding has been unable to meet the technical requirements at the present stage, and it is imperative to find a new type of cable-stayed cantilever scaffolding cantilever fixed beam.

\section{Insufficient and hidden dangers of traditional cable-stayed cantilever scaffolds}

Traditional cantilever scaffolds are embedded in beams and cast-in-place floors by hoops. They can not be embedded on PC laminated panels and directly affect the construction of buildings ${ }^{[2]}$. In addition, there are hidden dangers of external wall leakage, external frame safety, steel consumption and structural safety. 


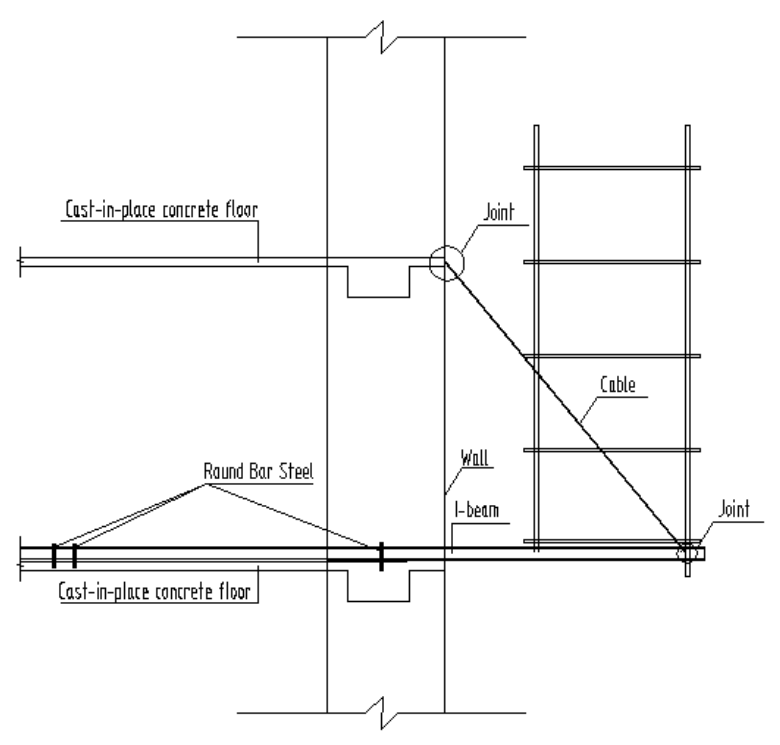

Fig. 1: The diagram of Traditional cantilevered scaffolding

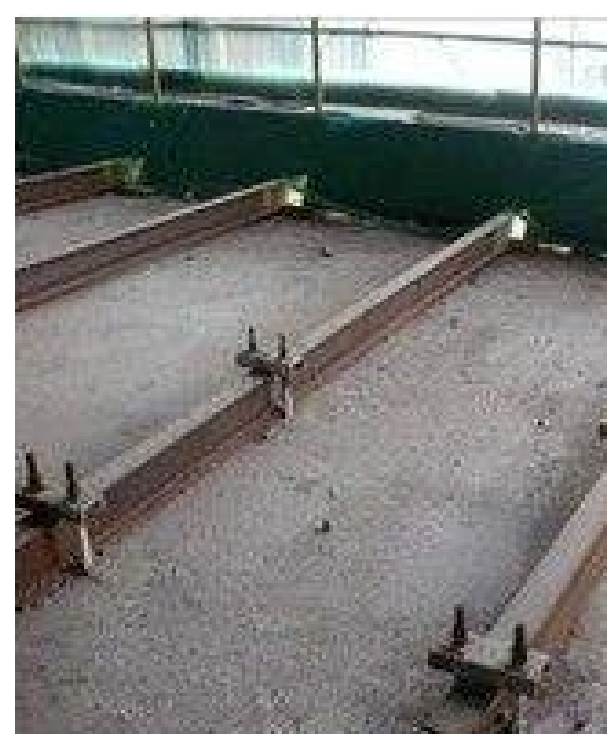

Fig. 2: Site construction photos

\section{External wall leakage}

The cantilever beam of the traditional cantilever scaffolding must be passed through the wall, and the cantilever I-steel is put on the floor. After the external scaffold is dismantled, it will leave the hole left by the I-steel through the wall, and it must be repaired after the outer frame is dismantled.If you pass through the shear wall, the I-steel in the shear wall will cause damage to the concrete when the local preembedding part will be damaged when the concrete is poured, and the cast steel can not be separated from the concrete after the demolition of the concrete. The only way is to cut it out and the I-steel in the concrete will remain in the shear wall forever. This will be the only way to leave the

I-steel in the concrete. The hidden danger of external wall leakage;Second, after the outer frame is dismantled, the I-steel hole can be repaired. It can not be sealed and vibrated from the outside. It can only be filled from the inside of the building. The repair work is relatively complex and complicated. The treatment of the grass roots is not in place. It is not in line with the loose and water to meet the requirements. If the repair work group is not strict or the responsibility is not good, the repair worker is repaired. If the work is complicated, the follow-up management and supervision are not in place, and the quality of repair work is lax, it may leave hidden danger of seepage. More individual teams and groups use mortar filling and brick filling phenomenon, which is also a hidden danger of water seepage.

\section{Improve the safety performance of external cantilever scaffolding}

The traditional cable-stayed suspension scaffolding is pulled by wire rope, and the wire rope has a fine elastic tension after the force is subjected to force. The cantilever scaffolding of the high-rise building is used for a long time, accumulating elastic tension for a long time, making the external cantilever scaffolding I-steel tilting forward, hanging the front section of the I-steel, and producing a certain safety of the scaffolding. The influence of the overhanging scaffold has great potential safety hazard.In addition, because the traditional cantilever scaffolding of I-steel must be put through the wall, the part will have a great influence on the position of the distribution tendons. Some even cut out the distribution tendons for the pre burial, and have a certain influence on the integrity of the shear wall and the brick masonry and the quality of the main structure.

After the removal of the outer frame of the I-beam through the wall, the method of post filling concrete placement is adopted. Subsequent repair will cause the tamping is not dense, the quality of raw materials can not be effectively guaranteed, and even use mortar or other materials to fill up.The main factors that affect the quality of the main body are the improper handling of the interface, the uncleanly cleaning at the base, the unloosening of the original concrete and the use of dry brick filling in the brick masonry. Through the wall I-beam affects the integrity of the main 
structure and brick masonry, resulting in poor quality of subsequent main body and brick masonry construction.

\section{Steel amount for cantilever scaffolding}

The traditional cantilevered scaffold steel I-beam is hung by internal hanging method [3]. The ratio between inside and outside cantilevers is generally 1.25:1.In other words, the internal shelving of Isteel is more than $50 \%$ of the total use of I-steel. The amount of steel material is larger, the material loss rate is high, and the reuse rate is relatively low, which can not achieve the effect of energy saving and emission reduction, and the degree of energy waste can be imagined.

\section{Reduction of repair costs and overall defects after the removal of the outer frame}

Through the wall I-beam affects the integrity of the main structure and brick masonry, resulting in poor quality of subsequent main body and brick masonry construction. The embedment of the through wall I-beam has a great influence on the location of the distribution reinforcement of the shear wall, and even the distributed bars will be cut off locally.It has a great influence on the structure of the main concrete shear wall, and the effect of filling material quality and poor quality on the quality of the structure is also affected by the damage of the material and manpower caused by the subsequent mending holes.

\section{Comparison of bottom anchored cable-stayed suspension scaffolding and its application}

The bottom anchor cable-stayed suspension scaffold is embedded in the casing, fixed on the structural beam or other component with anchor bolt. The bolt is connected with the bolt. It will not form the irregular hole, leaving only the casing hole of 25 diameter of the buried casing, the outer frame using the pull rod, the 22 round steel in the pull up and the middle use of the basket bolt in the middle, so that all the pulling is made. The rod is subjected to uniform force to improve the safety performance (Figure 3,4).

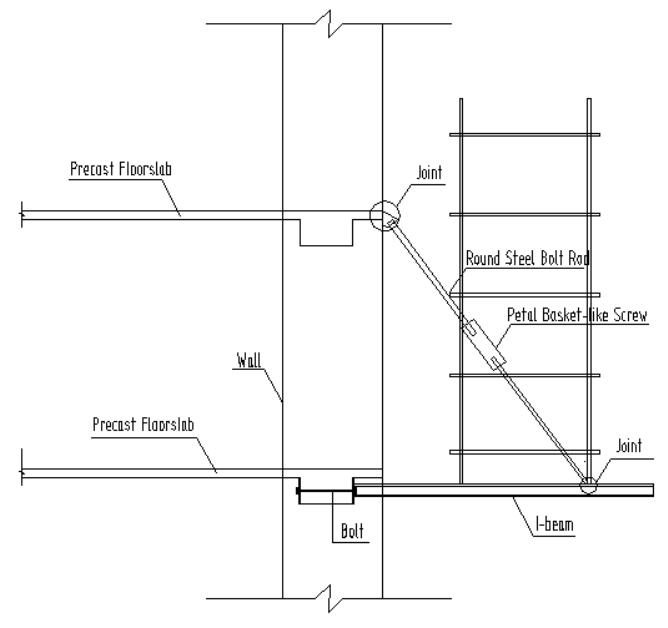

Fig. 3: The diagram of bottom anchor scaffold

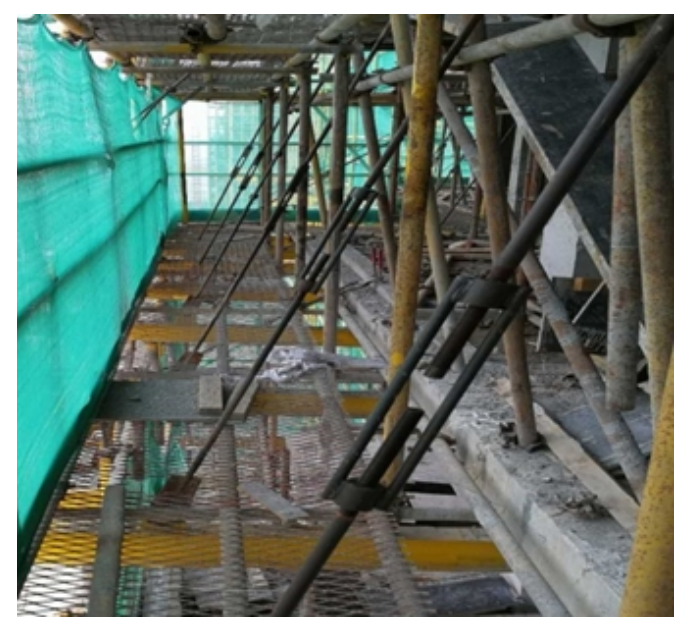

Fig. 4: Site construction photos

The use of bottom anchor cable-stayed cantilevered scaffold solves the problem of inembedment of PC laminated panels in building assembly.And through the field comparison, the choice of bottom anchorage cantilever scaffold is more than the traditional cantilever scaffolding. It also solves the water seepage of the exterior wall, the repair work of the subsequent hole, the safety of the main body structure, the safety performance of the outer frame itself, the utilization of steel and so on.

The original traditional cantilever scaffolding part of the wall I-steel is damaged by the pre buried template or other material in the concrete pouring, causing the I-steel to be dismantled from the condensing concrete. It must be cut out of the I-steel, and a large number of I-steel will be destroyed and no effective use can be used.It has a great influence on the reuse of raw materials of I-steel, and has formed a vicious cycle of the use of I-steel materials at one time. At the same time, the use of bottom anchor cable-stayed scaffold can greatly improve the efficiency of steel turnover. 
After the traditional cantilever scaffold is removed from the external frame of the I-beam through the wall, the method of post filling concrete placement is adopted. Subsequent repair will cause the tamping is not dense, the quality of raw materials can not be effectively guaranteed, and even use mortar or other materials to fill up.The bottom anchor cable-stayed suspension scaffold adopts the method of burying casing and bolt fastening, and the subsequent repair only needs to use foam glue to fill the hole of the pre buried casing, and then finish the surface treatment.The concrete and block which can save the material to repair holes greatly save the labor cost and material cost, and effectively eliminate the hidden danger of external wall leakage.

Using bottom anchored cable-stayed suspension scaffolding, the anchor bolt and beam are connected to the beam without wall setting, increasing the overall effect of the structure, and reducing the greater deviation of the structural steel, thus greatly improving the construction quality and the quality of the structure.

The cantilever scaffold is one of the most dangerous sub item projects in the project implementation process. To ensure the safety and stability of the outer frame is the key of the project implementation process. The outer frame is pulled by the pull rod to improve the safety performance. The traditional cantilever scaffolding is pulled by wire rope, because the long time of the cantilever scaffolding is long, the fine elastic tension after the steel wire is subjected to force, the long time accumulation, the deformation of the cantilever scaffolding, leading to the greater safety and safety of the external cantilever scaffolding. Using the bottom anchor cable-stayed suspension scaffolding, the pulling adopts 22 round steel hoisting and the middle use of the flower basket bolt in the middle. The elastic tension is basically eliminated after all the pull rod is uniformly loaded, and the phenomenon of the forward tilt of the I-steel is basically solved.The safety of scaffolding itself has been greatly improved, which has fundamentally solved the hidden danger of safety.

The use of bottom anchor cable-stayed cantilevered scaffold greatly reduces the repair cost and labor cost after the demolition of the external frame.After the traditional cantilever scaffolding is dismantled, the interface of the repair site is not handled properly, the cleaning of the base level is not clean, the original concrete loose treatment is not thorough, the brick masonry part is filled with dry brick and so on.Moreover, the traditional cantilever scaffold holes have a certain effect on the main structure, so that the structure can not be formed at one time. The bottom anchor cable-stayed cantilever scaffolding is connected with bolt, which is convenient to dismantle. After the removal of the outer frame, it only needs to be filled with foam agent to block and form a solid connection. It is convenient to dismantle and not destroy the original material.

\section{Effect}

The total area of the three phase headquarters building of the village reconstruction project in the central business district of a city is 136157.9 square meters, with a height of 78.1-83.9m and 1-2 floors, including two floors of the main building block and 25-27 floors on the ground. Frame shear wall structure, assembly rate of $25 \%$, the main components are standard floor and staircase.After the base - anchor cable-stayed scaffolding is used in the headquarters building, the construction unit compares the bottom anchor cantilever scaffolding with the traditional scaffolding, and finds that the steel steel used for steel steel is 55\% less than that of the traditional cantilever scaffold, and the bottom anchorage external scaffold basically solves the hidden danger of water seepage.In the construction process, there is no need to leave the I-steel through the wall hole, and the repair work is reduced greatly after the demolition. The repair work is 8 times less than that of the traditional cantilever scaffold. The effect of the bottom anchor cable-stayed scaffold is $80 \%$ lower than that of the traditional cable-stayed scaffold for the safety of the structure. The leakage caused by external walls saves more than 40 million yuan in economic efficiency.Compared with the traditional cantilever scaffold, the bottom anchor cable-stayed scaffold also basically solves the problem of passing through the shear wall and affecting the structural safety hazard. Moreover, the safety performance of the bottom anchored cable-stayed scaffold cantilevered I-beam is higher than that of the traditional cantilever scaffold. 


\section{Reference:}

[1] Yang Zhenxin. Application of cable-stayed cantilevered scaffold technology in high-rise construction [J]. building safety.2014 (6): 8-12.

[2] People's Republic of China Ministry of housing and urban and rural construction. Construction construction fastener type steel tube scaffolding safety technical regulations: JGJ130-2011[S]. Beijing: China Construction Industry Press, 2011.

[3] People's Republic of China Ministry of housing and urban rural construction. Safety inspection standards for building construction: JGJ59-2011[S]. Beijing: China Construction Industry Press, 2011. 